\title{
What Really Matters for Social Adaptation Among Left-Behind Children in China? A Systematic Review and Meta-Analysis
}

\author{
Junhua Zhang ${ }^{1, *}$, Yu Zhang ${ }^{1} \&$ Fang $\mathrm{Xu}^{1}$ \\ ${ }^{1}$ School of Education Science, Jiangsu Key Laboratory for Big Data of Psychology and Cognitive Science, Yancheng \\ Teachers University, Yancheng, China \\ *Correspondence: School of Education Science, Jiangsu Key Laboratory for Big Data of Psychology and Cognitive \\ Science, Yancheng Teachers University, 50 Kaifang Road, Yancheng City, Jiangsu Province 224002, China. E-mail: \\ junhuazh2003@163.com
}

Received: August 14, 2019

Accepted: September 14, 2019 Online Published: October 17, 2019

doi:10.5430/wje.v9n5p41

URL: https://doi.org/10.5430/wje.v9n5p41

\begin{abstract}
Compared with non-left-behind children, left-behind children in China have lower social adaptation and the underlying reasons deserve further study. This systematic review and meta-analysis included 29 studies published between 2006 and 2019. Protective factors of Left-behind children's social adaptation were resilience $(r=0.574)$, self-efficacy $(r=0.538)$, emotional intelligence $(r=0.421)$, self-esteem $(r=0.404)$, positive coping $(r=0.471)$, attachment $(r=0.354)$ and social support $(r=0.338)$ while risky factors were loneliness $(r=-0.453)$ and social anxiety $(r=-0.360)$. Age, birthplace, father/mother migration and the frequency of parent-child communication also have a certain impact on their social adaptation, but the effect size is relatively small. This study can provide some enlightenment for intervention programs and policy adjustment. More empirical studies focusing on influencing factors for social adaptation of left-behind children will be needed in the future.
\end{abstract}

Keywords: left-behind children, meta-analysis, social adaptation, predictor

\section{Introduction}

Social adaptation refers to the capacity to compromise, relate, confront and to cooperate with the environment and others(Samadi \& Sohrabi, 2016). Social adaptation is of great significance to individuals and is closely related to various developmental outcomes, such as academic achievement, future job, life quality and physically and mentally healthy(Neely-Prado, Navarrete, \& Huepe, 2019).

With rapid development of social economy, the number of rural-urban migration is huge in the past several decades in China. Numerous children are left in the countryside by their migrant parents who go to cities for better income. Depending on the data from the Ministry of Civil Affairs of the People's Republic of China, there are more than 6.97 million rural Left-Behind Children in 2018(Li, 2018). Due to a long separation from parents, left-behind children may not be adequately monitored, thus hindering the development of their social adaptation. A recent meta-analysis found that social adaptation of left -behind children was significantly lower than non-left-behind children (Zhang, Yan, Qiu, \& Dai, 2018). Thus, a detailed understanding of influencing factors of social adaptation is necessary to change this disadvantage.

Various factors affecting the social adaptation of left-behind children are constantly being explored, including gender, grade, number of parents out, parenting styles, social support, coping styles, mental health, and many other factors. These factors are primarily from family, school, individual and society (Zhang, 2014). Family factors are considered to be primary factors influencing social adaptation of left-behind children. Parents and children constitute a family triangle structure in the reproductive system (Fei, 2007). Left-behind children are in the state of separation from parents for a long time. The stable family triangle structure has been broken and the family ecosystem is unbalanced when parents migrate. Supervision and education toward left-children have been reduced, which is risk of social adaptation(Sun \& Sun, 2019). Family atmosphere and its dimensions had a significant effect on left-behind children's mental health (Yang \& Liu, 2017). School-related factors are also given an important influence on social adaptation. Supportive school climate had a positive effect on left-behind while controlled school climate had a 
negative effect on them(Wang, 2018). Teachers support, peer support has a positive effect on left-children' social adaptation, while the sense of school belonging played a mediating role(Wei et al., 2016). It was found that a closer relationship with peers can lead to more active social interaction (Liang, 2017). Social support was negatively correlated to externalizing problem behavior and internalizing problem behavior (Zhao, Ling, Chen, \& Teng, 2017). Community environment, social support and perceived discrimination are essential social factors. In a rural community, the unsatisfactory environment and the humble quality of the educator affect seriously the left-behind children's social adaptation (Wang, 2007). Perceived discrimination negatively affected one's social adaptation(Yang et al., 2019). Individual-related factors are also crucial. Self-esteem is positively correlated with social adaptation, while loneliness is negatively correlated with social adaptation (Jin, 2011). It was pointed out that the resilience had a significant predictive power for their adaptation, which also provided a new perspective for improving the social adaptation of left-behind children (Liu, 2012). Liu and other scholars have conducted reviews of social adaptation among left-behind children, and reached a more general conclusion (Liu, 2008). However, there are some conflicting conclusions and it is difficult to determine which factors are most significant from these studies. This systematic review and meta-analysis aimed to summarize predictors of social adaptation among left-behind children, which may have potential implications for research and practice.

\section{Methods}

Recommendations from the Preferred Reporting Items for Systematic Reviews and Meta-Analyses (PRISRM) were followed in this study (Liberati et al., 2009).

\subsection{Search Strategies}

We searched CNKI, Wanfang database, Chongqing VPN database, PubMed (Medline), OVID database and Web of Knowledge on August 15, 2019. Search strategies and the number of documents were listed in Supplementary Material 1.The search items in CNKI was as follows:

\# 1 left-behind

\# 2 Children or adolescents

\# 3. Social adaptation or social Adaptation or Social Adaptation Ability

Searching: 1 and 2 and 3

\subsection{Inclusion and Exclusion Criteria of Literature}

Inclusion criteria: a) social adaptation was measured by any standardized scales; b) the same influencing factor was studied by more than two studies; 3 ) providing $r$ or other parameters that can be converted into $r$, such as $t, p$, etc.; 4) left-behind children in mainland China; (5) literature language is English or Chinese; and (6) no intervention measures are used. In the case of papers using the same data, we link them together.

Exclusion criteria: 1) no quantitative measurement of social adaptation; 2) the subjects are not left-behind children in the mainland; 3) case studies. After screening, the influencing factors mainly focus on gender, grade, left-behind characteristics, self-esteem, self-efficacy, social support, peer relationship and so on. We divide them into demographic predictors, individual predictors, and contextual predictors.

\subsection{Literature Screening and Data Extraction}

We imported all documents into endnote and used "Find Duplicates" to remove duplicates. Then two researchers read the titles and abstracts of the non-repetitive papers independently and excluded irrelevant literature. After that, two researchers downloaded the full PDFs and read them independently. They removed the non-conforming references and list the reasons for exclusion. For the retained studies, two authors independently extracted the data. The main data extracted included: first author, publication date, survey area, number of subjects, age of subjects, measurement scales, influencing factors, main conclusions, gender of subjects, correlation coefficient or other parameters that can be converted into $r$, etc.

\subsection{Outcomes}

The main outcome indicators are the correlation between social adaptation (total score or sub-dimension) and various factors. When $r$ is not given in the literature, $t$ and which can be converted to $r$, are used. When both positive adaptation and negative adaptation are included in studies, only positive adaptation data is extracted. 


\subsection{Study Quality Checklist}

The same study quality check was used as a previous study (Chai, Du, Li, Su, \& Lin, 2019). There were 14 items in the checklist, including reporting, external validity, internal validity and power. Higher score meant higher quality of methodology. Two authors independently rated score of each included study and reached consensus through further discussions. The content of the checklist was listed in Supplementary Material 2.

\subsection{Statistical Analysis}

Pearson correlation coefficient was the effect size in this meta-analysis. For studies without $r$ but with inferential statistical data (such as $t, F$ or $p$ ), we use Comprehensive Meta-Analysis 3.0 software to integrate them into $r$. When comparing three or more groups, we compare each category in pairs. For example, types of going out can be divided into three categories (father going out alone, mother going out alone and both parents going out). Pearson correlation coefficient $r$ was calculated through $t$, which can be computed by $m, s d$ and $\mathrm{n}$ in any two groups (Borenstein, Hedges, Higgins, \& Rothstein, 2011). For the years of parents going out and the grades of left-behind children, $r$ was calculated by the same method, using two groups at both ends because they are continuous variables. We use Cohen's guidance to explain the effect size, where $r$ is at least $0.10=$ small, $0.30=$ medium, and $0.50=$ large (Cairns, Yap, Pilkington, \& Jorm, 2014; Cohen, 1992).Because of the heterogeneity among the studies, we use the random effect model to analyse the data (Cortese et al., 2018). $\mathrm{I}^{2}$ was used to assess heterogeneity while Egger test and funnel plots to assess publication bias (Egger, Davey Smith, Schneider, \& Minder, 1997; Egger, Smith, \& Phillips, 1997).

\section{Results}

\subsection{Characteristics of Included Studies}

556 non-repetitive references were screened in this study. The PRISMA flow chart in Figure 1 detailed the number of references selected and rejected at each stage.Reasons for rejection after screening the full text can be found in Supplementary Material 3. The final included research can be found in Supplementary 4. Children's attitudes towards parents migration, race, parenting style, number of peers, sports lifestyle, family social-economic status, teacher-student relationship, peer relationship, interpersonal self-reliance, alienation, health quality, peer communication ability, locus of control, social problem solving ability, self-control, discrimination perception, sense of responsibility, gratitude, depression, family intimacy and sense of security were excluded because only one study was available.

Table 1 listed author, year, number, region, measurement of social adaptation, and influencing factors. A total of 15702 left-behind children were included with 103 effective values. The subjects involved 18 provinces, including Anhui, Chongqing, Fujian, Guangxi, Guizhou, Hebei, Henan, Hubei, Hunan, Jilin, Jiangxi, Liaoning, Shandong, Shanxi, Shanghai, Sichuan, Yunnan and Zhejiang. The mean score of study quality was 11/14, ranging from 8 to 13 . However, most studies scored zero in terms of representation of sampling. 


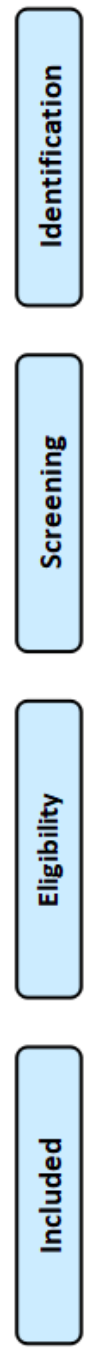

Records identified through database searching ( $n=546$ )
Additional records identified through other sources

( $n=10)$
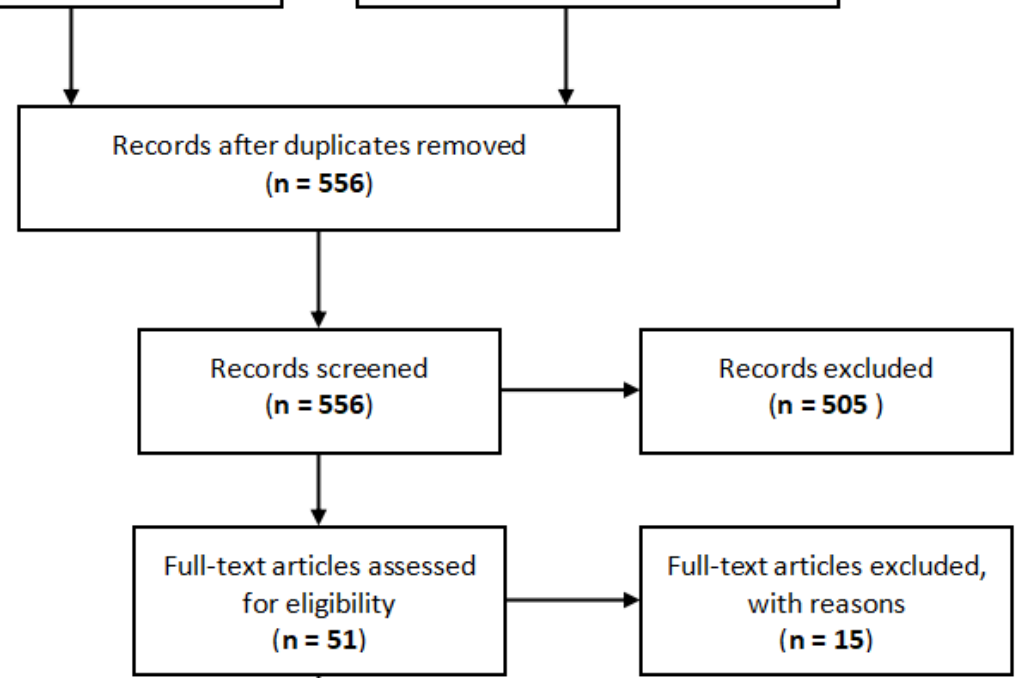
qualitative synthesis ( $n=36$ )

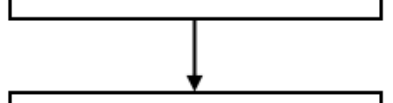

Studies included in quantitative synthesis (meta-analysis)

( $\mathbf{n}=\mathbf{2 9}$ datasets, reported in $\mathbf{3 6}$ references)

Figure 1. Preferred Reporting Items for Systematic Reviews and Meta-Analyses

Table 1. Descriptive Table of Studies Included

\begin{tabular}{|c|c|c|c|c|c|c|c|}
\hline $\begin{array}{l}\text { Author/ } \\
\text { Year }\end{array}$ & $\mathrm{n}$ & $\begin{array}{l}\text { Grade/ } \\
\text { Age }\end{array}$ & Region & Scales of social adaptation & $\begin{array}{l}\text { Number of } \\
\text { effect size }\end{array}$ & predictors & Quality \\
\hline $\mathrm{Bi}, 2015$ & 36 & G7-9 & Liaoning & $\begin{array}{c}\text { Social adaptation of second } \\
\text { school students by Chen } \\
\text { J.W }\end{array}$ & 3 & grade, gender, self-esteem & 10 \\
\hline Chen, 2018 & 715 & G10-11 & Henan & $\begin{array}{c}\text { Adolescent Behavior } \\
\text { Questionnaire by Cui L.X }\end{array}$ & 3 & $\begin{array}{l}\text { father attachment, mother } \\
\text { attachment, peers attachment }\end{array}$ & 12 \\
\hline Cheng, 2018 & 882 & $<16 \mathrm{Y}$ & $\begin{array}{l}\text { Sichuan, } \\
\text { Yunnan, } \\
\text { Guizhou }\end{array}$ & $\begin{array}{c}\text { Social adaptation of second } \\
\text { school students by Chen } \\
\text { J.W }\end{array}$ & 7 & $\begin{array}{l}\text { gender, only-child, birthplace, } \\
\text { grade, care-giver, number of } \\
\text { parents migration, frequency of } \\
\text { parents going back }\end{array}$ & 9 \\
\hline Cui, 2009 & 1048 & G 3-6 & $\begin{array}{l}\text { Hunan,Anh } \\
\text { ui, } \\
\text { Shanghai, }\end{array}$ & $\begin{array}{c}\text { Social adaptation Scale by } \\
\text { Yang Y.P. }\end{array}$ & 2 & gender, self-esteem & 13 \\
\hline
\end{tabular}


Shandong,

Henan,Sha

nxi,

Zhejiang

$\begin{array}{cccc}\text { Deng, 2010 } & 400 & \text { G 7-8 } & \begin{array}{r}\text { Chongqin } \\ \text { Sichuan }\end{array} \\ \text { Gong, 2016 } & 600 & \text { G 7-9 } & \text { Jiangxi } \\ \text { Gu, 2012 } & 825 & \text { G 7-9 } & \text { Jilin } \\ & & & \\ \text { Guo, 2014 } & 122 & \begin{array}{c}4-6 \\ \text { years }\end{array} & \text { Hebei }\end{array}$

Kuang, $2013 \quad 267$

Li H, $2015 \quad 459 \quad$ G 7-9

Guizhou

Guangxi

\begin{tabular}{|c|c|c|c|}
\hline Li P, 2010 & 342 & G 3-7 & Guangxi \\
\hline Li SL, 2016 & 1416 & G $1-6$ & Chongqing \\
\hline Li ZQ, 2015 & 587 & G 7-9 & Sichuan \\
\hline Liu H, 2012 & 195 & $\begin{array}{c}>8 \\
\text { years }\end{array}$ & Hubei \\
\hline Liu ML, 2009 & 1016 & G 7-9 & $\begin{array}{c}\text { Chongqing, } \\
\text { Yunan }\end{array}$ \\
\hline Liu XJ, 2016 & 318 & G 4-9 & Anhui \\
\hline Liu XW, 2018 & 780 & G 7-9 & $\begin{array}{l}\text { Hunan, } \\
\text { Guangxi }\end{array}$ \\
\hline Pan, 2018 & 316 & G 6-9 & Guangxi \\
\hline
\end{tabular}

$\begin{array}{cccc}\text { Shen, 2010 } & 465 & \text { G5-6 } & \text { Hubei } \\ & & & \\ \text { Sun, 2018 } & 415 & \text { G7 } & \text { Unclear } \\ \text { Wang, 2012 } & 513 & \text { G 7-12 } & \text { Henan }\end{array}$

$\begin{array}{ccc}\text { Xiao, } 2015 \quad 1110 \quad \text { G4-9 } & \begin{array}{c}\text { Guangxi } \\ \text { Hunan }\end{array}\end{array}$

Social adaptation of second school students by Chen

$$
\text { J.W }
$$$$
\text { self-edited }
$$

Adolescent prospective adaptation questionnaire by

$$
\text { Mu PJ }
$$

Social Adaptive Behavior

Scale for Children Aged

$$
\text { 3-7 by Hangzhou }
$$

University

Social adaptation scale by

$$
\text { Jia.L.B }
$$

Social adaptation diagnostic scale by Zeng R.C

Social adaptation Scale by Yang Y.P.

Social adjustment disorder from Mental Health Rate Scales for Pupil

Adolescent social adaptation behavior scale

$$
\text { by Zou } \mathrm{H} \text {. }
$$

Adolescent Psychological Adaptation Scale

Social adaptation of second school students by Chen

$$
\text { J.W }
$$

Adolescent social adaptation behavior scale by Zou H.

Social adaptation scale by $\mathrm{Hu} \mathrm{T}$.

Social adaptation Scale by Yang Y.P.

Social adaptation scale by Hu T. self-edited self-edited
2

positive coping, negative coping

gender

resilience

loneliness

self-esteem, resilience

12

only-child, class cadre, gender, grade, self-esteem, emotional intelligence,number of parents migration

gender, emotional intelligence

gender, grade

gender, grade, social anxiety

gender, class cadre, only-child gender, grade, care-giver, frequency of parents-child communication, years of parents migration, father attachment, mother attachment, peers attachment

gender, grade, frequency of parents-child communication, years

of parents migration, number of 


\begin{tabular}{|c|c|c|c|c|c|c|c|}
\hline & & & & & & $\begin{array}{l}\text { parents migration, social support, } \\
\text { resilience }\end{array}$ & \\
\hline Xie, 2014 & 641 & G 7-9 & Hunan & $\begin{array}{l}\text { Social adaptation of second } \\
\text { school students by Chen }\end{array}$ & 5 & $\begin{array}{c}\text { gender, resilience, positive coping, } \\
\text { negative coping, self-efficacy }\end{array}$ & 11 \\
\hline Yan,2016 & 290 & G7-9 & Hunan & $\begin{array}{c}\text { J.W } \\
\text { Social adaptation of second } \\
\text { school students by Chen }\end{array}$ & 5 & $\begin{array}{l}\text { gender, grade, father attachment, } \\
\text { mother attachment, peers }\end{array}$ & 12 \\
\hline & & & & J.W & & attachment & \\
\hline Yu,2013 & 232 & G 7-9 & Anhui & $\begin{array}{l}\text { Adolescent social } \\
\text { adaptation behavior scale }\end{array}$ & 2 & social support, resilience & 10 \\
\hline Zeng,Y 2017 & 331 & G7-9 & $\begin{array}{l}\text { Hubei,Fujia } \\
\text { n }\end{array}$ & $\begin{array}{c}\text { by Zou H. } \\
\text { Adolescent social } \\
\text { adaptation behavior scale }\end{array}$ & 2 & emotional intelligence, self-esteem & 11 \\
\hline $\begin{array}{c}\text { Zhang,GL, } \\
2017\end{array}$ & 684 & G7-9 & Anhui & $\begin{array}{c}\text { by Zou H. } \\
\text { Social adaptation Scale by } \\
\text { Yang Y.P. }\end{array}$ & 3 & gender, grade, loneliness & 11 \\
\hline $\begin{array}{l}\text { Zhang,MM, } \\
2012\end{array}$ & 413 & G 7-8 & Anhui & $\begin{array}{l}\text { social maladjustment scale } \\
\text { from MMPI }\end{array}$ & 2 & $\begin{array}{c}\text { social anxiety, number of parents } \\
\text { migration }\end{array}$ & 11 \\
\hline $\begin{array}{c}\text { Zheng,HF } \\
2009\end{array}$ & 284 & G4-8 & Henan & $\begin{array}{c}\text { Social adaptation Scale by } \\
\text { Yang Y.P. }\end{array}$ & 1 & $\begin{array}{l}\text { frequency of parents-child } \\
\text { communication }\end{array}$ & 12 \\
\hline
\end{tabular}

Table 2. Meta-Analysis of Demographic Predictors (r)

\begin{tabular}{|c|c|c|c|c|c|c|c|c|c|c|c|}
\hline \multirow[t]{2}{*}{ Type of analysis } & \multirow[t]{2}{*}{$\mathrm{K}$} & \multirow[t]{2}{*}{$\mathrm{N}$} & \multirow[t]{2}{*}{$\mathrm{r}$} & \multirow[t]{2}{*}{$95 \% \mathrm{CI}$} & \multirow[t]{2}{*}{$\mathrm{Z}$} & \multirow[t]{2}{*}{$\mathrm{P}$} & \multicolumn{3}{|c|}{ Heterogeneity } & \multicolumn{2}{|c|}{ Egger's Test } \\
\hline & & & & & & & Q & $\mathrm{p}$ & $\mathrm{I} 2$ & $\mathrm{t}$ & $\mathrm{p}$ \\
\hline gender & 18 & 11087 & -0.070 & $-0.159,0.020$ & 1.535 & 0.125 & 375.777 & 0.000 & 95.476 & 1.130 & 0.274 \\
\hline Grade/age & 13 & 6246 & 0.060 & $0.035,0.085$ & 4.754 & 0.000 & 71.937 & 0.000 & 83.319 & 0.513 & 0.617 \\
\hline only-child & 3 & 1756 & -0.033 & $-0.085,0.019$ & 1.235 & 0.217 & 2.433 & 0.296 & 17.787 & 6.543 & 0.096 \\
\hline Class cadre & 2 & 874 & 0.264 & $0.160,0.361$ & 4.839 & 0.000 & 2.636 & 0.104 & 62.062 & & \\
\hline birthplace & 2 & 1762 & 0.081 & $0.034,0.127$ & 3.393 & 0.001 & 0.868 & 0.352 & 0.000 & & \\
\hline care-giver & & & & & & & & & & & \\
\hline $\begin{array}{l}\text { care-giver(parents-grandp } \\
\text { arents) }\end{array}$ & 5 & 2641 & -0.007 & $-0.052,0.038$ & 0.309 & 0.757 & 5.310 & 0.257 & 24.665 & 0.277 & 0.799 \\
\hline care-giver(parents-others) & 5 & 1678 & -0.014 & $-0.064,0.036$ & 0.558 & 0.577 & 4.239 & 0.375 & 5.648 & 1.340 & 0.272 \\
\hline care-giver(father-mother) & 3 & 520 & -0.033 & $-0.172,0.108$ & 0.454 & 0.650 & 4.791 & 0.083 & 59.764 & 2.565 & 0.236 \\
\hline number of parents migration & & & & & & & & & & & \\
\hline $\begin{array}{l}\text { father migration } \\
\text { only-mother migration } \\
\text { only }\end{array}$ & 5 & 1835 & -0.017 & $-0.062,0.029$ & 0.710 & 0.478 & 3.129 & 0.536 & 0.000 & 0.039 & 0.971 \\
\hline Both parents & 6 & 4435 & -0.030 & $-0.059,-0.00$ & 1.998 & 0.046 & 3.580 & 0.622 & 0.000 & 1.354 & 0.247 \\
\hline $\begin{array}{l}\text { migration-one parent } \\
\text { migration }\end{array}$ & & & & 1 & & & & & & & \\
\hline years of parents migration & 4 & 1388 & -0.007 & $-0.113,0.100$ & 0.124 & 0.901 & 9.820 & 0.020 & 69.450 & 0.229 & 0.839 \\
\hline $\begin{array}{l}\text { frequency of parents going } \\
\text { back }\end{array}$ & 2 & 415 & 0.047 & $-0.050,0.143$ & 0.954 & 0.340 & 0.892 & 0.345 & 0.000 & & \\
\hline $\begin{array}{l}\text { frequency of parents-child } \\
\text { communication }\end{array}$ & 4 & 1813 & 0.115 & $0.005,0.223$ & 2.040 & 0.041 & 15.274 & 0.002 & 80.358 & 0.051 & 0.963 \\
\hline
\end{tabular}


Table 3. Meta-Analysis of Individual Predictors (r)

\begin{tabular}{|c|c|c|c|c|c|c|c|c|c|c|c|}
\hline \multirow[t]{2}{*}{ Type of analysis } & \multirow[t]{2}{*}{$\mathrm{K}$} & \multirow[t]{2}{*}{$\mathrm{N}$} & \multirow[t]{2}{*}{$\mathrm{r}$} & \multirow[t]{2}{*}{$95 \% \mathrm{CI}$} & \multirow[t]{2}{*}{ Z } & \multirow[t]{2}{*}{$\mathrm{P}$} & \multicolumn{3}{|c|}{ Heterogeneity } & \multicolumn{2}{|c|}{ Egger's Test } \\
\hline & & & & & & & Q & $\mathrm{p}$ & $\mathrm{I}^{2}$ & $\mathrm{t}$ & $\mathrm{p}$ \\
\hline resilience & 6 & 3354 & 0.574 & $0.484,0.652$ & 10.187 & 0.000 & 62.244 & 0.000 & 91.967 & 0.722 & 0.510 \\
\hline self-efficacy & 2 & 1421 & 0.538 & $0.470,0.601$ & 12.785 & 0.000 & 3.109 & 0.078 & 67.839 & & \\
\hline emotional intelligence & 3 & 1128 & 0.421 & $0.103,0.661$ & 2.546 & 0.011 & 68.892 & 0.000 & 97.097 & 0.461 & 0.724 \\
\hline self-esteem & 7 & 3206 & 0.404 & $0.092,0.645$ & 2.496 & 0.013 & 512.293 & 0.000 & 98.829 & 1.519 & 0.189 \\
\hline positive coping & 3 & 1526 & 0.471 & $0.431,0.510$ & 19.938 & 0.000 & 0.183 & 0.913 & 0.000 & 3.030 & 0.202 \\
\hline negative coping & 3 & 1526 & -0.116 & $-0.390,0.176$ & 0.770 & 0.436 & 67.079 & 0.000 & 97.018 & 3.274 & 0.188 \\
\hline loneliness & 2 & 888 & -0.453 & $-0.504,-0.400$ & 14.525 & 0.000 & 0.347 & 0.556 & 0.000 & & \\
\hline social anxiety & 2 & 304 & -0.360 & $-0.477,-0.230$ & 5.180 & 0.000 & 0.011 & 0.915 & 0.000 & & \\
\hline
\end{tabular}

Table 4. Meta-Analysis of Contextual Predictors (r)

\begin{tabular}{|c|c|c|c|c|c|c|c|c|c|c|c|}
\hline \multirow[t]{2}{*}{ Type of analysis } & \multirow[t]{2}{*}{$\mathrm{K}$} & \multirow[t]{2}{*}{$\mathrm{N}$} & \multirow[t]{2}{*}{$\mathrm{r}$} & \multirow[t]{2}{*}{$95 \% \mathrm{CI}$} & \multirow[t]{2}{*}{ Z } & \multirow[t]{2}{*}{$\mathrm{P}$} & \multicolumn{3}{|c|}{ Heterogeneity } & \multicolumn{2}{|c|}{ Egger's Test } \\
\hline & & & & & & & Q & $\mathrm{p}$ & $\mathrm{I}^{2}$ & $\mathrm{t}$ & $\mathrm{p}$ \\
\hline Social support & 4 & 2644 & 0.338 & $0.192,0.470$ & 4.369 & 0.000 & 43.710 & 0.000 & 93.137 & 0.077 & 0.945 \\
\hline Attachment & 3 & 1518 & 0.354 & $0.107,0.560$ & 2.763 & 0.006 & 50.871 & 0.000 & 96.096 & 0.672 & 0.623 \\
\hline father attachment & 3 & 1518 & 0.324 & $0.083,0.530$ & 2.604 & 0.009 & 47.202 & 0.000 & 95.763 & 0.349 & 0.785 \\
\hline mother attachment & 3 & 1518 & 0.358 & $0.103,0.570$ & 2.704 & 0.007 & 54.455 & 0.000 & 96.327 & 0.379 & 0.769 \\
\hline peers attachment & 3 & 1518 & 0.311 & $0.070,0.518$ & 2.505 & 0.012 & 46.690 & 0.000 & 95.716 & 0.637 & 0.638 \\
\hline
\end{tabular}

\subsection{Demographic Predictors}

According to table 2 and Supplementary Material 5, age, birthplace, class cadre, one/both parent migration and frequency of parents-child communication have significant influence on social adaptation of left-behind children. Left-behind children from towns score higher than left-behind children from villages. Older left-behind children and class cadre score higher than others. Left-behind children with low frequency of parent-child communication have lower social adaptation. However, according to Cohen's criteria, they are all less than 0.3 , which mean a small amount of effect size. Other factors including gender, only-child, care-giver type have no significant influence.

\subsection{Individual Predictors}

According to Table 3, individual variables include self-esteem, resilience, emotional intelligence, self-efficacy, coping style (positive coping, negative coping), social anxiety, loneliness. Except negative coping in coping style, other variables have a significant impact on social adaptation of left-behind children. Self-esteem, resilience, emotional intelligence, self-efficacy, positive coping are protective factor of social adaptation. According to Cohen's criterion, the correlation coefficients of resilience and self-efficacy with social adaptation are greater than 0.5 , which are large effect value. The correlation coefficients of self-esteem, emotional intelligence and positive coping with social adaptation is between 0.3 and 0.5 , which are medium effect value. The correlation coefficient between social anxiety and loneliness and social adaptation is less than 0 , which are risk factors of social adaptation. According to Cohen's criterion, the absolute value of correlation coefficient is between 0.3 and 0.5 , which belongs to the medium effect value.

\subsection{Contextual Predictors}

According to Table 4, the external factors include social support, attachment (father attachment, mother attachment and peer's attachment), which are the protective factor of social adaptation. According to Cohen's criterion, the correlation coefficient of social support, attachment and social adaptation is between 0.3 and 0.5 , which belongs to the moderate effect value.

\section{Discussions}

This study examined what factors influenced social adaptation of Chinese left-behind children and some key protective factors and risk factors were identified across 29 studies.

The effect of all demographic variables is a small effect value, indicating little influence on social adaptation, partly 
because most studies are not randomly sampled. The biggest predictor of demographic variable is class cadre. Those on duty in class have higher social adaptation, which implies that providing students with certain responsibilities in class is positively related to their social adaptation. Some uncontrollable variables such as gender, only-child, and age have little guidance for educational interventions.

Individual variables are most important predictors of social adaptation while strongest predictors were resilience and self-efficacy with large effect sizes in the current review. Individuals with high resilience can achieve relatively good development results after serious adversity(Rutter, 2013). Self-efficacy has much potential for playing a major role in helping one gain successful experience (Maddux \& Stanley, 1986). The results suggest the importance of enhancing resilience and self-efficacy in the process of improving social adaptation among Chinese left-behind children.

As to contextual predictors, attachment and social support were found to have medium effect sizes in this study, consistent with previous studies (Chai et al., 2019; Oldfield, Humphrey, \& Hebron, 2016). Parent attachment can help children explore unfamiliar environments and promote interaction with others. The acceptance and trust of peers are the foundation of stable social skills and are helpful in reducing the sense of alienation. Participation in collective activities or frequent exchange of ideas can increase the level of parent and peer attachment. Social support not only helps a person to achieve a goal, but also helps to share emotions, frustrations or to reach an understanding of problems (Cullen, 1994). Consistent with social connection theory (Hirschi, 1969), previous studies have found the protective effect of peer support and resilience on social adaptation of left-behind children (Lan \& Wang, 2019). Peer relationship is beneficial to children's mental health and social adaptation (Rubin, Bukowski, \& Laursen, 2009), and Intervention programs focusing on enhancing resilience and peer relationship can help improve social adaptation of left-behind children. According to the theory of social support, individuals with more social support are likely to maintain psychological health in the face of stress (Hobfoll, Freedy, Lane, \& Geller, 1990). Therefore, helping left-behind children to gain more perceived or actual social support from the community and social networks is an important means to improve their social adaptation.

These conclusions need to be treated with caution for some shortcomings. Firstly, all the studies included were all cross-sectional. Strictly speaking, these studies focused on the correlation between variables rather than causality. Secondly, they deal with the relationship between two variables without paying attention to the complex relationship among multiple variables. Thirdly, some conclusions only depend on a few empirical studies (even only two) due to the number limit of studies included, which may reduce the reliability and stability of the study. In addition, there may be some differences on classification of predictors and different classification may lead to different consequences.

\section{Conclusion}

This study focuses on predictors of social adaptation, which may deepen our understanding of the social adaptation among Chinese left-behind children. Demographic variables including number of parent migration and care-giver type had little influence on the social adaptation of Left-behind children. Resilience, self-efficacy, attachment, social support, positive coping, loneliness and social support were the top seven protective predictors. Social anxiety and loneliness are important risk factors. The results of our meta-analysis should be taken into account when formulating interventions aimed at strengthening social adaptation. More high-quality studies in this field are needed in future studies.

Authors Contributions: JZ conceived and supervised this study.YZ and FX screened the references and extracted the data.All authors read and approved the final manuscript.

Funding: This study was supported by National Social Science Fund of China (17BSH097).

Availability of Data and Materials: The data sets analyzed during the current study is available from the corresponding author on reasonable request.

\section{References}

Borenstein, M., Hedges, L. V., Higgins, J. P. T., \& Rothstein, H. R. (2011). Introduction to meta-analysis \| when does it make sense to perform a meta-analysis? 357-364. https://doi.org/10.1002/9780470743386.ch40

Cairns, K. E., Yap, M. B. H., Pilkington, P. D., \& Jorm, A. F. (2014). Risk and protective factors for depression that 
adolescents can modify: A systematic review and meta-analysis of longitudinal studies. Journal of Affective Disorders, 169, 61-75. https://doi.org/10.1016/j.jad.2014.08.006

Chai, X., Du, H., Li, X., Su, S., \& Lin, D. (2019). What really matters for loneliness among left-behind children in rural China: A meta-analytic review. Frontiers in Psychology, 10. https://doi.org/10.3389/fpsyg.2019.00774

Chen, X. (2015). Who can lighten left-behind children's lamp of soul? psychological problems analysis of left-behind children's suicide event in bijie city of Guizhou Province. Youth and Children Research and Practice, 14(4), 24-28.

Cohen, J. (1992). A power primer. Psychological Bulletin, 112(1), 155-159. https://doi.org/10.1037/0033-2909.112.1.155

Cortese, S., Sun, S., Zhang, J., Sharma, E., Chang, Z., Kuja-Halkola, R., . . . Faraone, S. V. (2018). Association between attention deficit hyperactivity disorder and asthma: a systematic review and meta-analysis and a $\begin{array}{lllll}\text { Swedish population-based } & \text { study. }\end{array}$ https://doi.org/10.1016/s2215-0366(18)30224-4

Cullen, F. T. (1994). Social support as an organizing concept for criminology: Presidential address to the academy of criminal justice sciences. Justice Quarterly, 11(4), 527-559. https://doi.org/10.1080/07418829400092421

Egger, M., Davey Smith, G., Schneider, M., \& Minder, C. (1997). Bias in meta-analysis detected by a simple, graphical test. Bmj, 315(7109), 629-634. https://doi.org/10.1136/bmj.315.7109.629

Egger, M., Smith, G. D., \& Phillips, A. N. (1997). Meta-analysis: principles and procedures. British Medical Journal, 315(7121), 1533-1537. https://doi.org/10.1136/bmj.315.7121.1533

Fei, X. (2007). The local China. Shanghai: Shanghai Century Publishing Group.

Hirschi, T. (1969). Causes of Delinquency. Berkeley: University of California Press.

Hobfoll, S. E., Freedy, J., Lane, C., \& Geller, P. (1990). Conservation of social resources: social support resource theory. Journal of Social and Personal Relationships, 7(4), 465-478. https://doi.org/10.1177/0265407590074004

Jin, X. (2011). The relationship among self-esteem, loneliness and social adaptation of left-behind children in rural areas. Paper presented at the The 14th National Psychological Conference, Xi'an.

Lan, X. Y., \& Wang, W. C. (2019). Direct and interactive effects of peer support and resilience on psychosocial adjustment in emerging adults with early left-behind experiences. Psychology Research and Behavior Management, 12, 277-288. https://doi.org/10.2147/prbm.s202774

Li, Y. (2018). Ministry of civil affairs: There are more than 6.97 million rural left-behind children in China. Retrieved from http://politics.gmw.cn/2018-08/31/content_30902762.htm

Liang, F. (2017). A Study on the relationship between attachment and social adaptation of rural Left-behind junior middle school students. Contemporary Education Science, (02), 93-96. https://doi.org/10.3969/j.issn.1672-2221.2017.02.023

Liberati, A., Altman, D. G., Tetzlaff, J., Mulrow, C., Gotzsche, P. C., Ioannidis, J. P. A., . . Moher, D. (2009). The PRISMA statement for reporting systematic reviews and meta-analyses of studies that evaluate healthcare interventions: explanation and elaboration. Bmj-British Medical Journal, 339. https://doi.org/ARTNb270010.1136/bmj.b2700

Liu, H. (2012). Study on resilience and social adaptation of Left-behind Children.Central South University for China.

Liu, M. (2008). The influencing factors of social adaptation among left-behind junior high school students. Journal of Inner Mongolia Normal University, 21(10), 65-68.

Maddux, J. E., \& Stanley, M. A. (1986). Self-efficacy theory in contemporary psychology. Journal of Social and Clinical Psychology, 4(3), 249-255. https://doi.org/10.1521/jscp.1986.4.3.249

Neely-Prado, A., Navarrete, G., \& Huepe, D. (2019). Socio-affective and cognitive predictors of social adaptation in vulnerable contexts. Plos One, 14(6), 218-236. https://doi.org/10.1371/journal.pone.0218236

Oldfield, J., Humphrey, N., \& Hebron, J. (2016). The role of parental and peer attachment relationships and school connectedness in predicting adolescent mental health outcomes. Child and Adolescent Mental Health, 21(1), 
21-29. https://doi.org/10.1111/camh.12108

Ouyang, Z., \& Fan, X. (2018). Family SES and psychological capital and self-esteem among left-behind children. $\begin{array}{llllll}\text { Chinese } \quad \text { Journal } & \text { Psychology, } & \text { 26(6), } & 1182-1185 .\end{array}$ https://doi.org/10.16128/j.cnki.1005-3611.2018.06.028

Rubin, K. H., Bukowski, W. M., \& Laursen, B. (2009). Handbook of peer interactions, relationships, and groups. New York: Guilford.

Rutter, M. (2013). Annual Research Review: Resilience - clinical implications. Journal of Child Psychology and Psychiatry, 54(4), 474-487. https://doi.org/10.1111/j.1469-7610.2012.02615.x

Samadi, M., \& Sohrabi, N. (2016). Mediating Role of the Social Problem Solving for Family Process, Family Content, and Adjustment. Procedia-Social and Behavioral Sciences, 217, $1185-1188$. https://doi.org/10.1016/j.sbspro.2016.02.141

Sun, W., \& Sun, Y. (2019). Familial ecosystem: the construction path of care service system for rural left-behind children. Social Work and Management, 19(4), 56-65.

Wang, Q. (2007). On the affect of community environment on the socialization of the children left in countrysides. Journal of Qiqihar University, 36(9), 47-49. https://doi.org/10.3969/j.issn.1008-2638.2007.05.014

Wang, S. (2018). The effect differences of school climate on non-/left-behind children' emotional intelligence and policy enlightenment. Modern Educational Management, 38(4), 100-105. https://doi.org/10.3969/j.issn.1674-5485.2018.04.018

Wei, X., Yu, C., Zhao, C., Wang, Z., Liu, Y., \& Wang, J. (2016). The mediating role of sense of school belonging in school atmosphere and academic achievement of left-behind children Chinese Journal of School Health, 37(7), 1103-1105. https://doi.org/ 10.16835 /j.cnki.1000-9817.2016.07.043

Yang, C., \& Liu, J. (2017). Family atmosphere Influence on rural left-behind children's psychological health:only raising without educating? Psychological Exploration, 37(4), 364-368.

Yang, J., Liu, X., Zhao, F., Wang, L., Liu, X., Zhou, H., \& Shi, B. (2019). The Effects of Perceived Discrimination and City Identity on the Social Adaptation of Migrant Children in Public and Private Schools. Stress Health. https://doi.org/10.1002/smi.2869

Zhang, J., Yan, L., Qiu, H., \& Dai, B. (2018). Social adaptation of Chinese left-behind children: Systematic review and meta-analysis. Children and Youth Services Review, 95, 308-315. https://doi.org/10.1016/j.childyouth.2018.11.012

Zhang, L. (2014). Study on social adaptation of left-behind children in western rural areas. Nanjing University of Science and Technology.

Zhao, N., Ling, Y., Chen, Q., \& Teng, X. (2017). The influence of social support to rural left-behind children's problem behaviors: the mediating effects of hope. China Journal of Health Psychology, 25(8), 1227-1231. https://doi.org/10.13342/j.cnki.cjhp.2017.08.030

Zhou, C., Lv, Z., Xing, H., Yu, W., Chen, Y., \& Qian, Q. (2019). Study on quality of life , social support , and family parenting pattern and the corre- lations for left - behind children. Maternal and Child Health Care of China, 39(4), 887-890. https://doi.org/10.7620/zgfybj.j.issn.1001-4411.2019.04.54 